\title{
Contour integrals for numerical computation of discrete eigenvalues in the Zakharov-Shabat problem
}

\author{
Anastasila Vasylchenkova ${ }^{1,2, *}$, Jaroslaw E. Prilepsky ${ }^{1}$, and Sergei K. Turitsyn ${ }^{1}$ \\ ${ }^{1}$ Aston Institute of Photonic Technologies, Aston University, Birmingham, B4 7ET, UK \\ ${ }^{2}$ System Analysis Research Institute, Aston University, Birmingham, B4 7ET, UK \\ *Corresponding author: vasylcha@aston.ac.uk
}

Compiled July 12, 2018

\begin{abstract}
We propose a novel algorithm for the numerical computation of discrete eigenvalues in the Zakharov-Shabat problem. Our approach is based on contour integrals of the nonlinear Fourier spectrum function in the complex plane of the spectral parameter. The reliability and performance of the new approach are examined in application to a single eigenvalue, multiple eigenvalues and the degenerate breather's multiple eigenvalue. We also study the impact of additive white Gaussian noise on the stability of numerical eigenvalues computation. (C) 2018 Optical Society of America
\end{abstract}

OCIS codes: (070.4340) Nonlinear optical signal processing; 000.4430 Numerical approximation and analysis.

http://dx.doi.org/10.1364/ao.XX.XXXXXX

The Zakharov-Shabat (ZS) spectral problem [1] has attracted a lot of attention recently in the context of optical communications, see [2-4] and references therein. This problem can also be frequently encountered in coupled-mode theory, see [5] and references there, is important for various applications ranging from the design and fabrication of fibre Bragg gratings [6] to solving nonlinear equations via the so-called inverse scattering transform (IST) - original name for the nonlinear Fourier transform (NFT). The importance of the ZS problem is greatly enhanced by its relation to the integrability of the nonlinear Schrödinger equation (NLSE) - the master model for nonlinear science with a number of applications in physics, biology and engineering. Efficient numerical computation of the nonlinear spectra of the ZS problem is therefore important for various applications. We consider the case of the focusing NLSE, corresponding to anomalous dispersion of the optical fibre, where the associated ZS problem becomes non-Hermitian. Importantly, the anomalous dispersion NLSE supports the existence of bright solitons corresponding to discrete complex eigenvalues of the ZS problem [1].

The conventional ZS problem deals with the determination of the NFT spectrum corresponding to a complex "potential" $q(\tau)$ (e.g., optical signal), vanishing at $\tau \rightarrow \pm \infty$. In general, for the case of anomalous dispersion, the NFT spectrum consists of a continuous part (a complex function of the real-valued parameter $\xi$, where the latter can be understood as the nonlinear analogue of frequency) and a discrete part (complex eigenval- ues and related additional parameters, the so-called norming constants). The eigenvalues have positive imaginary part and describe the solitonic modes, while the continuous NFT spectrum refers to the radiative components [1-3]. The ZS problem is defined as a pair of coupled ordinary differential equations:

$$
\frac{d \phi_{1}}{d \tau}=-i \xi \phi_{1}+q(\tau) \phi_{2}, \quad \frac{d \phi_{2}}{d \tau}=-q^{*}(\tau) \phi_{1}+i \xi \phi_{2}
$$

written for two auxiliary functions $\phi_{1,2}(\tau, \xi)$. To determine the NFT spectrum, we need to study the particular solutions of the ZS problem (1) fixed through their asymptotes at $\tau \rightarrow-\infty$ : $\phi_{1}(\tau) \rightarrow e^{-i \xi \tau}, \phi_{2}(\tau) \rightarrow 0$. These special solutions of the ZS problem are called Jost functions [1]. (It also is possible to deal with Jost solutions fixed at $\tau \rightarrow \infty$ [2]). The ZS system involves a (generally complex) spectral parameter $\xi$, playing, as was noted before, the role of frequency. The core part of the NFT - the spectral functions (scattering coefficients) associated with our profile $q(\tau)$ - are defined through Jost solution asymptotes at $\tau \rightarrow \infty:$

$$
a(\xi)=\lim _{\tau \rightarrow+\infty} \phi_{1} e^{i \xi \tau}, \quad b(\xi)=\lim _{\tau \rightarrow+\infty} \phi_{2} e^{-i \xi \tau}
$$

and the continuous spectrum (reflection coefficient) $r(\xi)$ is given by the ratio: $r(\xi)=b(\xi) / a(\xi)$ for real $\xi$. The zeros of the spectral function $a(\xi)$ in the upper half-plane of $\xi$ represent solitonic eigenvalues, and the norming constants in case of simple zeros are the residues of $r(\xi)$ at its poles (when $b$ is analytic at these points). For our purposes it is important that $a(\xi)$ is an analytic function in the upper half-plane of $\xi$, while $b(\xi)$ is analytic in the region $\Im \xi>0$ only if $q(\tau)$ has a finite support [7].

In this Letter we focus on the numerical computation of discrete (solitonic) eigenvalues, i.e. on finding zeros of the function $a(\xi)$ from (2). The most common approach aiming at that, among the NFT-related works, implies the use of iterative methods $[2,3,8]$, the spectral method, based on the solution of block matrix eigenproblem [3], or the application of specifically optimised grid search in the upper half-plane of $\xi$ [9]. However, these approaches are not always reliable (e.g., in optical transmission applications the eigenvalues can be considerably corrupted by the optical noise $[2,10])$ and possess some drawbacks described below. Here we apply the contour integration method (the well-known Delves-Lyness zero-search algorithm [11]) for 
eigenvalues evaluation that mitigates some of the existing problems, especially in the case when the eigenvalues are settled in the close proximity of each other, or degenerate.

For the information transmission applications, the discrete eigenvalues of the $\mathrm{ZS}$ problem are often located inside a certain (usually known a priory) area in the complex $\xi$-plane, which is specified by the considered scheme. In optical communications, this zone in the complex $\xi$-plane can be defined by the encoding NFT alphabet [12], or can encompass "a band" in multiplexed NFT spectrum. We refer to this area as the region of interest (ROI). The principal advantage of the proposed method is that it locates all eigenvalues simultaneously, such that its complexity weakly depends on the number of eigenvalues inside the given ROI. The spectral and iterative methods do not limit any area of search by default, when in grid search approach, instead of computing the ZS problem solution (the $a(\xi)$-function) at each mesh node using some sufficiently fine ROI grid (red stars in Fig. 1), we just need to determine $a(\xi)$ values for the points along the boundary of ROI (green dots in Fig. 1). In contrast to iterative algorithms,

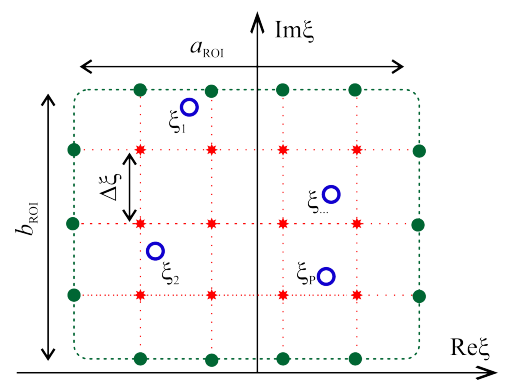

Fig. 1. Concepts of grid search and contour integrals for eigenvalues computation in the $\xi$-plane.

the performance of our method depends in a predefined way on the ROI size or sampling interval, and it is not affected by the accuracy of initial guess for an eigenvalue. The spectral method returns a lot of eigenvalues, whilst only a few of them are true components of discrete spectrum, therefore this method requires additional processing with larger complexity for expected higher accuracy. The contour integrals approach does not have this issue. These properties make the contour integrals a viable tool for multi-eigenvalue NFT communication [12-14].

An efficient method implementing the contour integrals (i.e. the quadrature-based method) for an analytic function zeros' search was described by Delves and Lyness [11]. According to their work, in order to find the eigenvalues, we define the set of contour integrals $\left\{s_{p}\right\}_{p=1}^{P}$ as

$$
s_{p}=\frac{1}{2 \pi i} \int_{C} \xi^{p} \frac{a^{\prime}(\xi)}{a(\xi)} \mathrm{d} \xi=\sum_{j=1}^{P} \xi_{j}^{p}, \quad p=1 \ldots P,
$$

where $C$ is a (simply connected) manually chosen contour in the complex $\xi$-plane, enclosing our ROI, and the prime designates the derivative with respect to $\xi$. The expression with $p=0$ gives the total number of eigenvalues $P$ inside $C$, accounting for their multiplicity (if we have any degenerate zeros). The evaluation of $\left\{s_{p}\right\}$ allows deriving the so-called Newton's identities $\left\{\sigma_{p}\right\}_{p=1}^{P}$ :

$$
\begin{aligned}
\sigma_{1} & =-\sum_{j=1}^{P} \xi_{j}, \\
\sigma_{2} & =\xi_{1} \xi_{2}+\xi_{2} \xi_{3}+\ldots+\xi_{P-1} \xi_{P}, \\
\ldots & \\
\sigma_{P} & =(-1)^{P} \xi_{1} \xi_{2} \ldots \xi_{P},
\end{aligned}
$$

which are linked to contour integrals $s_{p}$ as

$$
\begin{aligned}
s_{1}+\sigma_{1} & =0, \\
s_{2}+s_{1} \sigma_{1}+2 \sigma_{2} & =0, \\
\ldots & \\
s_{N}+s_{N-1} \sigma_{1}+\ldots+s_{1} \sigma_{N-1}+N \sigma_{N} & =0 .
\end{aligned}
$$

This system of equations can be solved recurrently using the values of $\sigma_{p}$ obtained in the previous iteration:

$$
\sigma_{p}=\frac{1}{p}\left(s_{p}+\sum_{j=1}^{p-1} s_{j} \sigma_{p-j}\right), \quad p=1 \ldots P .
$$

The same $\sigma_{p}$-s are (up to a sign) the Vieta's formulae for the following polynomial:

$$
M(z)=z^{P}+\sigma_{1} z^{P-1}+\sigma_{2} z^{P-2}+\ldots+\sigma_{P-1} z+\sigma_{P} .
$$

The polynomial $M(z)$ has exactly the same zeros as the initial function $a(\xi)$. Therefore, using any polynomial root-finding technique, the desired set of eigenvalues $\left\{\xi_{j}\right\}_{j=1}^{P}$ can be found.

The promising feature of the presented approach is its intuitive stability: all eigenvalues can be successfully located inside the desired ROI, while all spurious eigenvalues arising from the presence of either optical noise or numerical imperfections, typically appear outside the ROI and thus can be readily eliminated. Note also that the iterative and grid search algorithms cannot distinguish the simple and multiple zeros of a function unless additional procedures are embedded into the respective root-finding algorithm, though even in that case the algorithm can fail to produce the correct result. This might be especially important for NFT-based communication schemes operating with higher-multiplexity eigenvalues [15]. The contour integrals approach is free from the aforementioned problems: it evaluates the eigenvalues according to their multiplicity and is not affected by how close the eigenvalues are.

We would like to specifically stress the advantage of the proposed numerical algorithm in terms of the computational complexity (the estimated number of elementary operations involved). Compared to the grid search, using the notations of Fig. 1 with $\Delta \xi$ being a length of subinterval along the integration contour and $a_{\mathrm{ROI}}, b_{\mathrm{ROI}}$ being (rectangular) ROI dimensions, the complexity of the contour integrals method is $O\left(2\left(a_{\mathrm{ROI}}+b_{\mathrm{ROI}}\right) / \Delta \xi \cdot N_{\mathrm{ZS}}\right)$, while for the grid search the complexity is $O\left(a_{\mathrm{ROI}} b_{\mathrm{ROI}} / \Delta \xi \cdot N_{\mathrm{ZS}}\right)\left(N_{\mathrm{ZS}}\right.$ here is the number of operation to solve (1) for a single value of $\xi)$. This reduction can be substantial for a large ROI or a dense grid. We note that the numerical error of contour integrals approach is inversely proportional to the distance between the boundaries of ROI and the nearest zero [11]. So it is possible to improve the contour integrals approach accuracy by the larger factor than grid search improvement gaining less penalty in complexity. When using the additional iterative refinement to reach the acceptable eigenvalue accuracy, as it was done in $[7,9]$, we need to perform $O\left(a_{\mathrm{ROI}} b_{\mathrm{ROI}} / \Delta \xi \cdot N_{\mathrm{ZS}} \cdot P N_{\text {iter }}\right)$ operations. The iterative algorithm, implemented alone, requires $O\left(N_{Z S} \cdot P N_{\text {iter }}\right)$ operations, where $N_{\text {iter }}$ is the number of steps to reach the desired zero with a given accuracy. But when we have an insufficiently accurate initial guess, the number of required iterative steps $N_{\text {iter }}$ can be dramatically large, going to infinity when the algorithm cannot converge. The spectral method has complexity $\mathrm{O}\left(\mathrm{N}^{3}\right)$ for $N$-sample signal, and it requires additional processing to extract proper eigenvalues with unpredictable complexity. 
The conventional ZS problem solution methods require $O\left(N^{2}\right)$ $[3,16]$, though the spectral method requests rougher sampling for the same level of accuracy. Finally we note that the presented root-finding method can be straightforwardly paired with the fast NFT method [8], which reduces the complexity of signal processing, and of the eigenvalues search in our case, towards the fast Fourier transform-order.

The main parameter that impacts the accuracy of a given NFT method, is the temporal domain discretisation step $\Delta \tau$. Having in mind the optical communications application, we test the contour integrals approach using the sequence of profiles with randomly chosen eigenvalues from the given constellations that were suggested for NFT-based transmission [17]. Here we use two kinds of constellations depicted in inset of Fig. 3.

We examine the performance of the contour integrals approach for single solitons, 2-eigenvalues and 4-eigenvalues profiles. We use the Darboux method [4] to generate such states with the arbitrary chosen norming constants and compute the respective eigenvalue portrait in the perfect back-to-back scenario (deterministic case, Fig. 2) and in back-to-back adding a white Gaussian noise to the profile in the temporal domain, Fig. 3. As a metric of computational accuracy (for the computed eigenvalue $\xi$ from the initial one $\xi_{0}$ ), we use an error vector magnitude (EVM) defined as $\mathrm{EVM}^{2}=\left\langle\left|\xi-\xi_{0}\right|^{2}\right\rangle /\left\langle\left|\xi_{0}\right|^{2}\right\rangle$, where \langle\rangle designates the averaging over the sequence of profiles and over eigenvalues in the profiles. The normalisation over the mean value of constellation points appears after centring of the constellation diagram. For solving the ZS problem we use the popular Bofetta-

$\square$ contour integrals $\bigcirc$ iterative $\Delta$ grid search $\nabla$ spectral method

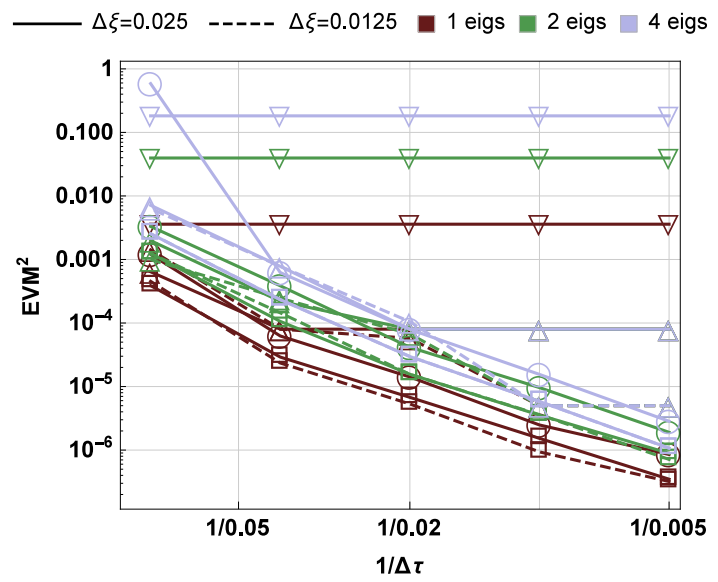

Fig. 2. $E M^{2}$ for noiseless profiles with different number of eigenvalues, contour sampling $\Delta \xi$ and numerical method with varying $\Delta \tau$. Results for the spectral method correspond to $N=51$ samples in the spectral domain.

Osborne method $[9,16]$, known to have second order accuracy and often superlative performance compared to other options [7]. For the strongly decaying signals used on our work we truncate them when $|q(\tau)|$ is close to zero. The signal is presented as a set of $N$ samples at the successive equidistant time moments: $q\left(\tau_{m}\right)=q_{m}$ for $\tau_{m}$ lying inside the symmetric finite interval $\left[-\tau_{N / 2}, \tau_{N / 2}\right]$, sampled with step size $\Delta \tau$. The discrete analogues of Jost functions, $\phi_{1,2}(\tau)$, are fixed at the left edge of the interval: $\Phi\left(-\tau_{N / 2}\right)=\left(\phi_{1}\left(-\tau_{N / 2}\right), \phi_{2}\left(-\tau_{N / 2}\right)\right)^{T}=\left(e^{i \tau_{N / 2}}, 0\right)^{T}$, and the $\tau$-evolution is performed by using the transfer matrix method [7]: $\Phi\left(\tau_{m+1}\right)=T_{m} \Phi\left(\tau_{m}\right)$. In our case, we employ the
Bofetta-Osborne transfer matrix $T_{m}[7,16]$. Contour integrals $s_{p}$ are computed using the trapezoidal rule along the contour. However, we notice that the error of the ZS problem solution $(a(\xi)$ evaluation at each $\xi$ ) is $O\left(\Delta \tau^{2}\right)$, while the contour integration is carried out inside the spectral domain giving the spectral error of the order of $O\left(\Delta \xi^{2}\right)$, such that choosing the consistent number of subintervals along the contour requires some further study. The back-to-back tests are performed for different temporal domain discretisation step $\Delta \tau$, and the tests for noise-corrupted profiles are performed studying the accuracy in dependence on the signal-to-noise ratio (SNR), defined as a fraction of signal and noise powers, irrelatively of the solitonic nature of our signals. The SNR value was altered through adding white Gaussian noise with varying noise power in the time domain.

In Figs. 2, 3 we predictably observe the reduction of EVM (i.e. of the method's error value) with the decrease of temporal domain discretisation step $\Delta \tau$ and with the increase of SNR. By changing the contour sampling $(\Delta \xi)$, we can manipulate the numerical accuracy of the contour integrals approach and get advantage comparably to the iterative method. The grid search and spectral methods display a higher error. The accuracy is typically better for the eigenvalues located along the imaginary axis (the constellation, corresponding to the filled circles in the inset of Fig. 3). The number of eigenvalues slightly influences the value of numerical error. One of the advantages of contour

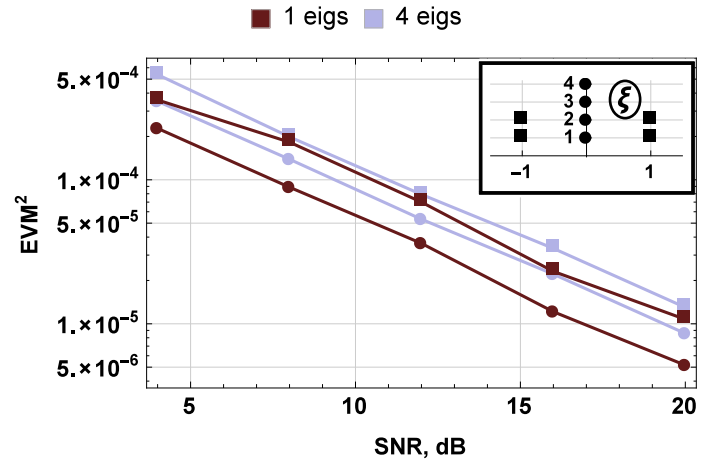

Fig. 3. $\mathrm{EVM}^{2}$ evaluation for different constellations (circles along the imaginary axis, squares - symmetrically around the imaginary axis) for a single eigenvalue and a 4-eigenvalue solution in dependence on SNR (temporal domain is sampled with $\Delta \tau=0.007$, contour is sampled with $\Delta \xi=0.0125)$.

integrals approach is its capability to easily evaluate a degenerate eigenvalue. We demonstrate this on the example of the degenerate breather [18]: a limiting case of two-eigenvalue soliton, when the eigenvalues approach each other. In the case of purely imaginary degenerate eigenvalue $\xi=i x$, we can use the profile given in [18]

$$
q_{\mathrm{deg}}(\tau)=\frac{8 i x(2 \tau x \sinh (2 x \tau)-\cosh (2 x \tau))}{8 x^{2} \tau^{2}+\cosh (4 x \tau)+1} .
$$

We expect to get two equal eigenvalues from the numerical algorithms, and it would mean that $a(\xi)$ has a double zero at that point. We observe that similarly to the non-degenerate case, the accuracy of grid search is worse than that for the iterative and contour integral approaches (see Fig. 4). Decreasing $\Delta \xi$, we can improve the accuracy for both grid search and contour integral algorithms. The error for contour integrals is generally 

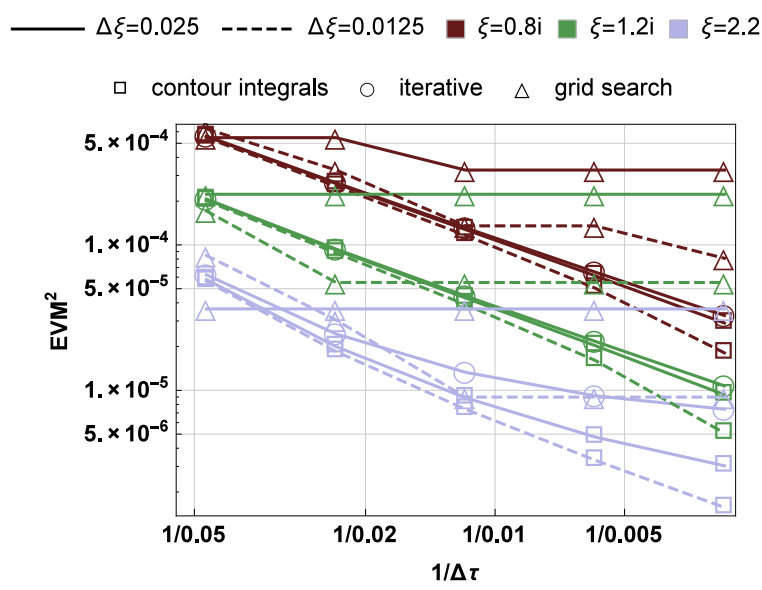

Fig. 4. $\mathrm{EVM}^{2}$ behaviour for degenerate eigenvalue evaluation via different numerical method, using noiseless degenerate breather profile Eq. (8), with the change of $\Delta \tau$.

smaller and degrades faster with the increase of $1 / \Delta \tau$ than the error for the iterative algorithm.

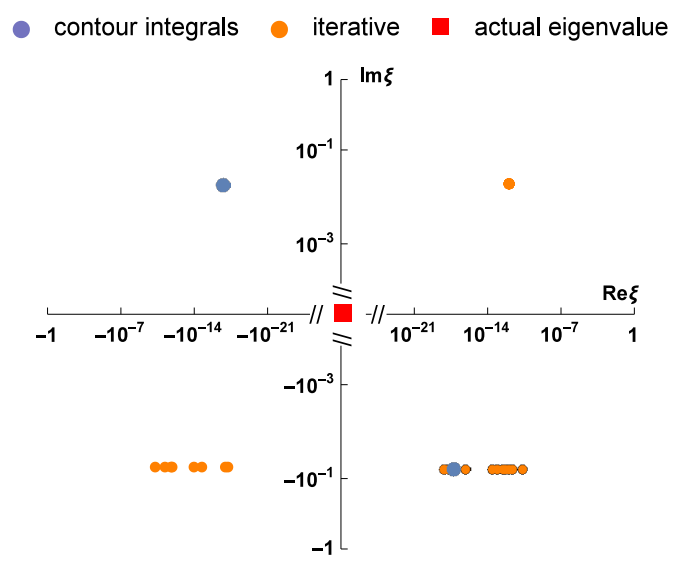

Fig. 5. Deviation of numerically evaluated eigenvalues of the noiseless degenerate breather profile $(\xi=1.2 i, \Delta \tau=0.023$, $\Delta \xi=0.0125)$, computed via contour integrals and via iterative method.

The actual behaviour of the contour integrals and iterative methods for the degenerate eigenvalues search is compared in Fig. 5 (the results for all eigenvalues are given in the coordinate system with the origin at the eigenvalue, the red square). None of the used numerical methods locate both eigenvalues at exactly the same point even for a noiseless profile. The contour integrals approach gives the symmetrical points around the actual eigenvalues, and their deviations are smaller than those for the iterative method. The initial guess point also influences the accuracy of iterative method's result, see the scattered points in the lower part of Fig. 5).

We also studied the performance of the contour integrals approach using the degenerate breather with added noise, in dependence on the SNR, Fig. 6. The results obtained are similar to those presented in Fig. 3 earlier.

However, one of the important findings of our paper is that the accuracy of the eigenvalues computation increases with the magnitude of the degenerate eigenvalue. The latter is related to the signal power. Therefore, we anticipate that the applicability

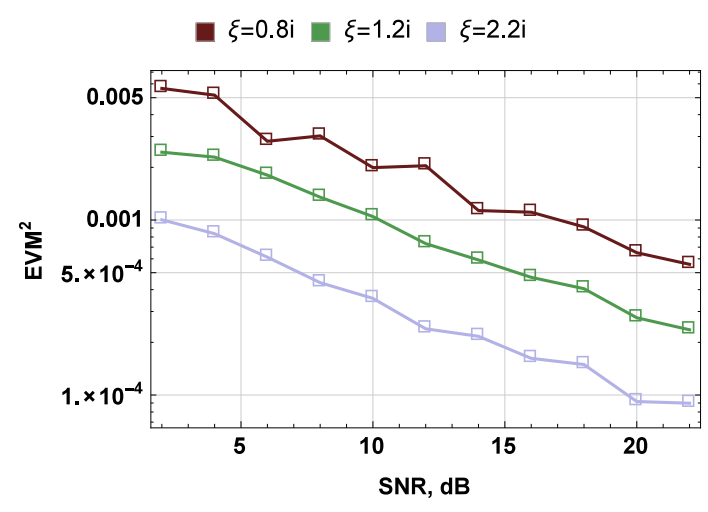

Fig. 6. $\mathrm{EVM}^{2}$ behaviour for the contour integrals method applied for the computation of eigenvalue corresponding to degenerate breather, with the change of SNR $(\Delta \xi=0.0125$, $\Delta \tau=0.003)$.

of the contour integrals approach will be even more important for the high power signal processing. The paramount goal of NFT processing is to operate in the highly-nonlinear regime where conventional linear techniques fail.

In this Letter we proposed and successfully tested a new numerical method for finding soliton eigenvalues, based on the computation of contour integrals. We demonstrated that even in the presence of noise, the accuracy of the proposed technique is only slightly affected by the number and multiplicity of eigenvalues. This feature can result in a substantial benefit for the NFTbased systems that use multiple eigenvalues or the degenerate eigenvalues. The method shows a rather small computational error for the degenerate and multi-eigenvalues "potentials" in comparison with the conventional iterative methods.

This work was partially supported by the Leverhulme Project RPG-2018-063.

\section{REFERENCES}

1. V. E. Zakharov and A. B. Shabat, Sov. Phys.-JETP 34, 62 (1972).

2. S. K. Turitsyn, J. E. Prilepsky, S. T. Le, S. Wahls, L. L. Frumin, M. Kamalian, and S. A. Derevianko, Optica 3, 307 (2017).

3. M. I. Yousefi and F. R. Kschischang, IEEE Transactions on Inf. Theory 60, 4346 (2014).

4. S. T. Le, V. Aref, and H. Buelow, Nat. Photon. 11, 570 (2017).

5. S. E. Miller, Bell Syst. Tech. J. 3, 661 (1954).

6. R. Kashyap, Fiber Bragg Gratings (Elsevier, 2010).

7. A. Vasylchenkova, J. E. Prilepsky, D. Shepelsky, and A. Chattopadhyay, preprint arXiv:1708.01144 (2017).

8. S. Wahls and H. V. Poor, Proc. Int. Conf. on Acoust. Speech, Signal Process. (ICASSP) pp. 5780-5784 (2013).

9. S. Burtsev, R. Camassa, and I. Timofeyev, J. Comput. Phys. 147, 166 (1998).

10. G. P. Agrawal, Nonlinear Fiber Optics (Academic Press, 2013).

11. L. M. Delves and J. N. Lyness, Math. Comp. 21, 543 (1967).

12. S. Hari, M. I. Yousefi, and F. R. Kschischang, J. Light. Technol. 34, 3110 (2016).

13. S. Hari and F. R. Kschischang, J. Light. Technol. 34, 3529 (2016).

14. T. Gui, C. Lu, A. Lau, and P. Wai, Opt. Express 25, 20286 (2017).

15. J. Garcia, preprint arXiv:1802.07456 (2018).

16. G. Boffetta and A. Osborne, J. Comput. Phys. 102, 252-264 (1992).

17. S. Hari, F. Kschischang, and M. Yousefi, 27th Biennial Symp. on Commun. (QBSC) pp. 92-95 (2014).

18. D. J. Kedziora, A. Ankiewicz, and N. Akhmediev, Phys. Rev. E 85 (2012).

19. E. A. Kuznetsov, Sov. Phys. Dokl. 22, 507 (1977). 